\title{
Lungenhochdruck
}

\section{Von Nitroglycerin zu innovativen Therapien}

— Riociguat (Adempas ${ }^{\circledast}$ ) ist ein gutes Beispiel für die Zusammenarbeit von Industrie und akademischen Institutionen bei der Entwicklung innovativer Therapien. An der Erforschung war ein Wis-

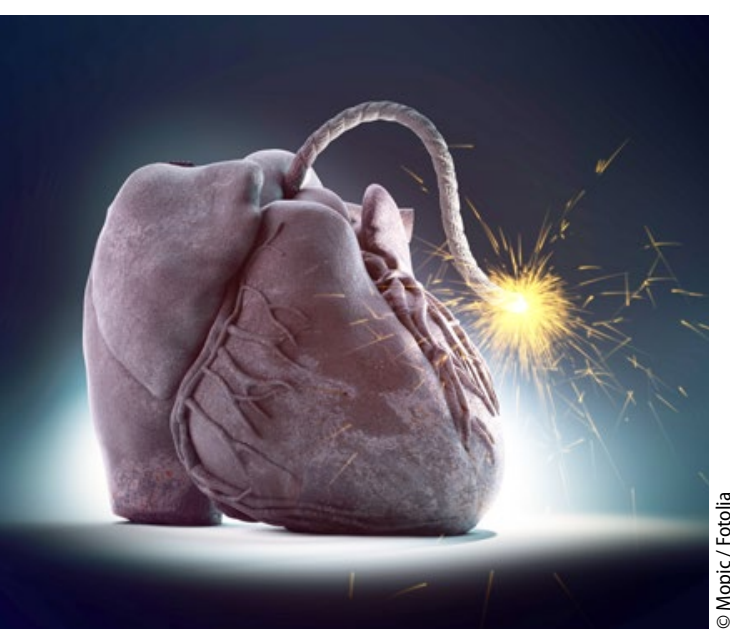

Die Zündschnur muss gekappt werden, bevor das Herz Schaden nimmt. senschaftlerteam der Firma Bayer und des Lungenforschungszentrums an der Justus-Liebig-Universität Gießen beteiligt, das im Jahr 2015 mit dem Deutschen Zukunftspreis ausgezeichnet wurde. Im Deutschen Museum in München wurde nun ein Ausstellungsmodul enthüllt, dass die Erforschung dieses Wirkstoffs modellhaft darstellt.

Anlässlich der Enthüllung des Moduls sagte Prof. Ardeschir Ghofrani, Oberarzt am Universitätsklinikum Gießen, der maßgeblich an der Entwicklung des Wirkstoffs beteiligt war: „Mit Riociguat ist es gelungen, nicht nur ein deutlich verfeinertes und viel potenteres $\mathrm{Ni}$ troglycerin herzustellen, sondern auch ein Medikament, das sehr spezifisch die Lungengefäße erweitert.“

\section{Entlastung für Herz und Lunge}

Riociguat ist die erste Substanz, die die lösliche Guanylat-Zyklase (sGC) stimu- liert. Außerdem erhöht es die Sensitivität der sGC für den endogenen Vasodilatator Stickstoffmonoxid (NO). Damit setzt Riociguat an einer pathomechanistischen Schlüsselstelle der pulmonalen Hypertonie an, die mit einem Mangel an endogenem NO und einer ungenügenden Stimulation der sGC einhergeht.

Zugelassen ist die Substanz zur Behandlung bestimmter Formen der chronisch-thromboembolischen pulmonalen Hypertonie (CTEPH) und pulmonal-arterieller Hypertonie (PAH). Die Studien CHEST-1 und PATENT-1 zeigen, dass Riociguat die körperliche Leistungsfähigkeit von Patienten mit PAH oder CTEPH anhaltend verbessert und dabei gut verträglich ist.

\section{Dr. Judith Neumaier}

- Pressekonferenz „Deutscher Zukunftspreis: Bekanntgabe der Nominierungen zur diesjährigen Preisvergabe"; München, September 2016 (Veranstalter: Bundespräsidialamt)

\section{Kurz notiert}

Indikationserweiterung für HPV-Impfstoff $\rightarrow$ Die europäischen Kommission hat den HPVImpfstoff Cervarix ${ }^{\oplus}$, einen Impfstoff gegen die humanen Papillomaviren der Typen 16 und 18, für die Indikation HPV-assoziiertes Analkarzinom zugelassen. Damit wird der Zugang beider Geschlechter zur Prävention von HPV-assoziiertem Analkarzinom in der Europäischen Union ermöglicht, und dies nicht nur für Erwachsene, sondern auch Mädchen und Jungen. Die Zulassung folgt auf die Entscheidung des Committee for Medicinal Products for Human Use der europäischen Arzneimittelzulassungsbehörde European Medicines Agency vom 23. Juni 2016. Klinische Studien hatten für Cervarix ${ }^{\circledast}$ Männern und Frauen eine vergleichbare Immunantwort gezeigt. AuBer in Europa ist der HPV-Impfstoff Cervarix ${ }^{\circledR}$ auch in anderen Ländern wie Chile, Kolumbien und Mexiko für die Prävention von HPV-assoziiertem Analkarzinom zugelassen.

Red.

- Nach Informationen von GSK

\section{Schwere Erkrankung - leichte Therapie}

\section{Schnelle Hilfe bei chronischer spontaner Urtikaria}

_ Juckende Quaddeln und/oder schmerzende Angioödeme sind typische Zeichen einer Urtikaria [Zuberbier T et al. Allergy. 2014;69:868-87]. Das Charakteristikum der chronischen spontanen Urtikaria (csU) sind die immer wiederkehrenden Symptome über mehr als sechs Wochen ohne erkennbare Ursache. Das schränkt die Lebensqualität der Betroffenen enorm ein. Die Beeinträchtigungen bei csU sind so stark, dass die körperlichen und seelischen Belastungen höher einzustufen sind als bei Psoriasis. Umso wichtiger ist die Behandlung der Betroffenen. Wird ein von csU betroffener Patient beim Allgemeinmediziner vorstellig, kann dieser durchaus selbst die Behandlung einleiten.

Im Gegensatz zur Schwere der Krankheitslast ist die Behandlung der $c s U$ vergleichsweise einfach: Gemäß der Leitlinie gelten $\mathrm{H}_{1}$-Antihistaminika $\left(\mathrm{H}_{1} \mathrm{AH}\right)$ als Standardtherapie [Zuberbier T et al. Allergy. 2014;69:868-87], jedoch sprechen nur etwa $50 \%$ der csU-Patienten ausreichend auf die zugelassene Dosierung an [Sánchez-Borges $\mathrm{M}$ et al. World Allergy Organ J. 2012; 5:125-47]. Selbst bei Hochdosierung der $\mathrm{H}_{1} \mathrm{AH}$ bis auf das Vierfache bleiben immer noch Patienten symptombelastet. Mit Omalizumab $\left(\right.$ Xolair $\left.^{\circledast}\right)$, das bei csU als Zusatztherapie alle vier Wochen in der Dosis von $300 \mathrm{mg}(2 \times 150 \mathrm{mg}$ zusätzlich zur Standardtherapie) verabreicht wird, kann csU-Patienten, die nicht ausreichend auf $\mathrm{H}_{1} \mathrm{AH}$ ansprechen, effektiv geholfen werden.

Red.

- Nach Informationen von Novartis 